\title{
Management of robotic bleeding complications
}

\author{
Pierluigi Novellis ${ }^{1}$, Mehmood Jadoon ${ }^{1}$, Umberto Cariboni ${ }^{1}$, Edoardo Bottoni ${ }^{1}$, Alessandro Pardolesi ${ }^{2}$, \\ Giulia Veronesi ${ }^{1}$ \\ ${ }^{1}$ Division of Thoracic Surgery, Humanitas Clinical and Research Center, Rozzano, Milan, Italy; ${ }^{2}$ Thoracic Surgery Unit, Fondazione IRCCS Istituto \\ Nazionale dei Tumori, Milan, Italy \\ Correspondence to: Pierluigi Novellis. Division of Thoracic Surgery, Humanitas Clinical and Research Center, Via Alessandro Manzoni 56,20089 \\ Rozzano, Milano, Italy. Email: pierluigi.novellis@cancercenter.humanitas.it.
}

Submitted Oct 23, 2018. Accepted for publication Jan 08, 2019.

doi: 10.21037/acs.2019.02.03

View this article at: http://dx.doi.org/10.21037/acs.2019.02.03

\section{Clinical vignette}

The minimally invasive approach in lung surgery is increasingly being used all over the world. There is reluctance from some surgeons to adopt the minimally invasive approach due to the risk of intraoperative complications that are sometimes more difficult to manage compared to the conventional open approach. The factors that determine risk of complication can be oncological factors such as the presence of a metastatic hilar lymph node attached to vascular branches and therefore a more complicated resection or the presence of fibrotic tissue because of a previous neoadjuvant treatment. Anatomic factors such as the absence of a complete fissure or the presence of fibrotic adhesions because of inflammation can also contribute to a difficult resection with high risk of complications. The foremost cause for conversion from a minimally invasive approach to a thoracotomy is bleeding, and the fear of bleeding is the main factor that discourages most surgeons to undertake a minimally invasive approach. Robotic surgery with its better visibility and maneuverability of instruments inside the chest could be considered a better tool to deal with complex surgical cases and to reduce the rate of conversion to open approach in the case of bleeding. However, intraoperative complications requiring conversions can also occur even with the robotic approach.

We present a series of five cases of pulmonary neoplasms which were treated with robotic approach and in which we encountered intraoperative complications, two of which required conversions.

\section{Case 1}

In the first video, we present the case of a 60 -year-old women with adenocarcinoma of the middle lobe T2N2M0. The patient underwent three cycles of induction chemotherapy with cisplatin and paclitaxel. Following an excellent response with reduction in the size of the paratracheal lymph node, she was scheduled for a robotic middle lobectomy with radical mediastinal lymphadenectomy.

\section{Case 2}

This case is a 72-year-old man suffering from early stage adenocarcinoma of the right upper lobe (T1bN0). The patient was a current smoker with history of COPD and hypertensive cardiomyopathy. He underwent a robotic right upper lobectomy with mediastinal lymphadenectomy.

\section{Case 3}

A 65-year-old man, ex-smoker with history of diabetes was diagnosed with a $2 \mathrm{~cm}$ carcinoid of the middle lobe during investigations for back pain. The patient was a candidate for a robotic middle lobectomy.

\section{Case 4}

The fourth case is a 43-year-old woman who presented to Accident and Emergency department for back pain. The CT scan of the chest revealed an aortic type B dissection and an incidental finding of $11 \mathrm{~mm}$ nodule of the middle lobe. Once the vascular problem was solved, the patient underwent a PET scan and subsequent biopsy of the nodule which proved this to be a lung adenocarcinoma. The patient was a candidate for a robotic middle lobectomy. 


\section{Case 5}

A 71-year-old smoker underwent a CT scan for thoracic trauma, with an incidental diagnosis of a $2 \mathrm{~cm}$ nodule of the right upper lobe associated with two further GGOs of the left lower lobe. The PET scan revealed an increased uptake of FDG at the level of the right upper lobe, and no uptake on the left side. The biopsy of the right upper lobe showed lung adenocarcinoma. The patient was a candidate for a robotic right upper lobectomy and follow-up was planned for the left sided nodules.

\section{Surgical technique}

The lung is isolated by double lumen endotracheal tube. The patient is placed in lateral position. An arm support and a bean bag are used to stabilize the patient on the table. Our surgical approach is the anterior 4 ports robotic technique (1). Four arms and four incisions are used. $\mathrm{CO}_{2}$ insufflation is not routinely used.

A $3 \mathrm{~cm}$ utility incision is performed in the fourth intercostal space anteriorly and a skin retractor (Alexys) is positioned. Camera port is placed in the seventh intercostal space along the anterior axillary line, and a 30-degree 3D endoscope is inserted to explore the thoracic cavity. Under direct vision, a $12 \mathrm{~mm}$ incision is performed in the same intercostal space along posterior axillary (this port is used to inset staplers thus $12 \mathrm{~mm}$ is needed) and the fourth $8-\mathrm{mm}$ incision is performed in the auscultatory triangle for the lung retractor.

We describe five intraoperative complications. The first three complications were successfully dealt with robotic approach, the last two cases required a conversion to open technique.

\section{Case 1 (middle lobectomy)}

After isolation and resection of middle lobe vein and bronchus with an anterior approach to hilum, the fissure was opened and the artery was isolated. During the isolation of one of the middle lobe arteries, an injury was made to middle lobe artery with the hook. A swab was placed and pressure was applied on the artery for 15 minutes. The anesthetist and theatre team were informed about pulmonary artery bleed. Fortunately, bleeding stopped and we were able to sling and staple the middle lobe artery and complete the operation without any further complications.

\section{Case 2 (right upper lobectomy)}

We started the dissection at the anterior hilum and we were moving superiorly towards the pulmonary artery. An injury to the first branch of the truncus anterior of the pulmonary artery was caused by inappropriate movement of the hook with subsequent copious bleeding. Fortunately the injury was distant from the origin of the branch from the main pulmonary artery. We were able to dissect and isolate the proximal part of the vessel which was subsequently slinged. A hem-o-lock clip was applied proximally and distally and the artery divided with scissors. The operation was completed with resection of the vein, the bronchus and the fissure using standard multiple endoscopic staplers.

\section{Case 3 (middle lobectomy)}

We were in the last stages of the operation and had already divided one middle lobe branch of the pulmonary artery, the pulmonary vein and the bronchus to the middle lobe. We found a second branch of the artery going to the middle lobe which was isolated and slinged. Bleeding was observed as the robotic stapler failed to properly staple. The PA branch was partially divided with distal half still intact. We were able to deal with this complication by applying a hem-o-lock to the pulmonary artery stump proximally which effectively controlled the bleeding and allowed us to complete the operation.

\section{Case 4 (middle lobectomy)}

The patient presented with a high body mass index and very raised diaphragm. Middle lobe vein was isolated. The introduction of the stapler was difficult because of a very high diaphragm. During the introduction of the stapler the curved bipolar dissector was inappropriately used to push down on the diaphragm and accidentally caused a trans-diaphragmatic injury to the liver. A sizable spurt of blood was seen coming through the diaphragmatic defect. Compression to the bleeding point was applied while the patient was converted to thoracotomy approach. Through a relatively small incision in the diaphragm cauterization of bleeding point was performed. Hemostasis was secured, a hemostatic sponge was placed on the liver surface and the diaphragmatic defect was closed. Middle lobectomy was completed with open approach. 


\section{Case 5 (right upper lobectomy)}

Dissection of the hilum was started anteriorly with isolation of the vein which was encircled with a vascular sling. During dissection of truncus anterior, one of the branch was accidently injured with the hook. Major bleeding was observed. Although bleeding was temporarily controlled, we were unable to safely manage the bleeding with keyhole approach. While the console surgeon was controlling the bleeding with the robotic approach, another senior surgeon was asked to convert to the open approach. Once thoracotomy was performed, bleeding was secured with vascular clamps, the pulmonary artery defect was repaired with a 5.0 suture and the right upper lobectomy was completed. The same patient underwent a robotic left lower lobectomy after 2 months for a synchronous second primary.

\section{Comments}

The robotic approach for lung cancer surgery has some particular advantages over the open approach $(2,3)$. Robotic approach for lobectomy and lymph node dissection is practicable, safe and associated with shorter postoperative hospitalization than open surgery. The number of lymph nodes removed also appears oncologically acceptable for early stage lung cancer (2). In other retrospective studies, a lower morbidity and mortality and an improved quality of life were observed in robotic group compared to thoracotomy group (3). Some initial data of a multicentric study showed that robotics is a safe and effective approach for selected locally advanced stages (IIIa) with good perioperative outcome and similar oncological results to that reported for open surgery (4).

In our series, we experienced the advocated advantages of the robotic approach: it is a comfortable approach for surgeon, it gives excellent three-dimensional vision, and there is absence of trembling, the immobility of the camera and the perfect reproducibility of the hand movements. All these factors make the robotic approach to the lung easier and more comfortable compared to VATS in particular for complex cases.

As with all minimally invasive approaches, there is always the risk of a vascular accident requiring a thoracotomy. The absence of tactile feedback, even if compensated by an excellent vision and a perfect three-dimensional perception, certainly constitutes a limitation of the current surgical robots. A further limitation is the presence of a rigid structure welded on the patient and the absence of the first operator at the operating table ready in sterile clothes. These factors obviously cause a delay in case of a vascular catastrophe.

However in our experience, vascular catastrophes have been very rare. Overall, our conversion rate was $6.2 \%$ (21 cases out of 338 major lung resections), of these 4 were due to bleeding $(1.1 \%)$. In most cases, the bleeding was controlled without modifying the approach. Conversion to open approach was required in only two cases. In liver bleeding, it was necessary to open the diaphragm and explore the liver dome in order to control the bleeding. One can argue that robotic approach could have been used to control bleeding from liver but it was not feasible because of the small size of the chest cavity due to very high position of the diaphragm. To avoid this complication, we should have pulled down the diaphragm, anchored it with a couple of stiches to the chest wall, before starting the resection, as we have done in other similar situations. In the fifth case, the vascular repair was not carried out with the robot because the vessel had a full-thickness tear and already a significant blood loss had occurred. In addition, we did not release the tension on the lung from the fourth arm that was retracting the lung posteriorly, increasing the amount of blood loss.

The advice we give in this regard is to always keep calm, control bleeding by compression, assess the situation, prepare for all possibilities and then slowly visualise the critical point. In case of necessary conversion utility incision can be used and converted to anterior thoracotomy approach, this allows the insertion of larger compression swabs or vascular clamps from the outside in a short time.

\section{Caveats}

* In case of minimal bleeding, compression for some minutes could solve the problem.

* Use the hook carefully, after the bleeding, presented in fifth video we have stopped using it for vascular dissection and we have started using the curved bipolar dissector.

* When the bleeding vessel is well isolated, a hem-o-lock could be used to control the bleeding.

* The robotic approach with two hands type of dissection, two graspers facilitates better control of the bleeding compared to VATS approach.

* Pay attention in short patient with a high BMI: in this case a high diaphragm and a short chest cavity can 
reduce the space for moving instruments. Consider using diaphragm retraction stich to chest wall.

* Where possible graspers could be used as a vascular clamps.

\section{Acknowledgements}

We thank Mehmood Jadoon for help with the English and Zaheer Raffeeq for being the narrator in the videos.

\section{Footnote}

Conflicts of Interest: G Veronesi has received honoraria from ABI Medica SpA, Medtronic and Verb Medical. The other authors have no conflicts of interest to declare.

Cite this article as: Novellis $\mathrm{P}$, Jadoon M, Cariboni U, Bottoni E, Pardolesi A, Veronesi G. Management of robotic bleeding complications. Ann Cardiothorac Surg 2019;8(2):292-295. doi: 10.21037/acs.2019.02.03

\section{References}

1. Park BJ, Melfi F, Mussi A, et al. Robotic lobectomy for non-small cell lung cancer (NSCLC): long-term oncologic results. J Thorac Cardiovasc Surg 2012;143:383-9.

2. Veronesi G, Galetta D, Maisonneuve P, et al. Four-arm robotic lobectomy for the treatment of early-stage lung cancer. J Thorac Cardiovasc Surg 2010;140:19-25.

3. Cerfolio RJ, Bryant AS, Skylizard L, et al. Initial consecutive experience of completely portal robotic pulmonary resection with 4 arms. J Thorac Cardiovasc Surg 2011;142:740-6.

4. Veronesi G, Park B, Cerfolio R, et al. Robotic resection of Stage III lung cancer: an international retrospective study. Eur J Cardiothorac Surg 2018;54:912-9. 